\title{
PROTOTIPO DE CONTROL DE ACCESO A AULAS Y REGISTRO AUTOMÁTICO DE ASISTENCIA
}

\section{PROTOTYPE OF A CLASSROOM ACCESS CONTROL AND AUTOMATIC ATTENDANCE RECORDS}

\author{
MSc. Luz E. Márquez C., Ing. Yesid N. Abdo L., Ing. Fernando J. Ángulo. \\ ITSA, Escuela TIC, Grupo de Investigación en Innovación y Tecnología GIIT \\ Calle 18, No 39-100, Soledad, Atlántico, Colombia. \\ Tel.: (+575) 3745911, Fax: (+575) 3746632. \\ E-mail: $\{$ lmarquez, ylara, fangulo\}@itsa.edu.co
}

\begin{abstract}
Resumen: El proyecto consiste en el diseño e implementación de un prototipo como prueba piloto, para el control de acceso a aulas y laboratorios, y registro automático de asistencia a clases, para reducir el tiempo que estas tareas conllevan. El proyecto inicia definiendo la estructura del sistema, luego se realizó un análisis de diferentes tecnologías que podrían implementarse. Posteriormente, se diseña el software adaptando la estructura operacional del sistema a los lineamientos y políticas requeridas, finalizando con el montaje de todas las etapas que constituyen el prototipo.
\end{abstract}

Palabras clave: Control de acceso, control de asistencia, Identificación por Radiofrecuencia, XBEE.

\begin{abstract}
This pilot project consists of the design and implementation of a prototype which is being built to control access to classrooms, laboratories, and automatic registration of attendance, to reduce the time that is involved in these processes. The project began by defining the structure of the system. Then, an analysis of the different technologies that could be implemented was conducted. After, the software was designed to adapt the system's operational structure to guidelines and institutional policies, ending with the assembly of all the phases that make up the prototype.
\end{abstract}

Keywords: Access Control, Attendance Control, Radio Frequency Identification, XBEE.

\section{INTRODUCCIÓN}

Las instituciones educativas cada vez más, se preocupan por implementar sistemas inteligentes de bajo costo y alta confiabilidad para el control de ingreso a sus instituciones y a ciertos espacios de los campus.

El mercado actual, y las empresas que a ello se dedican ofrecen diversos métodos de control de acceso, entre los que se destacan los lectores biométricos y los de radiofrecuencias.
En el Instituto Tecnológico de Soledad Atlántico ITSA, miembros de la institución en su papel de participantes activos por el desarrollo de su entorno y en especial de su propia institución, proponen una solución a la necesidad de control de ingreso a salones de clases y control automático de asistencia, implementando tecnología de punta que ofrezca flexibilidad, confiabilidad y economía.

La idea de proyecto surge debido a que el ingreso a las aulas de clases, salas de informática y laboratorios del instituto es un proceso netamente manual, que depende de una persona encargada de 
administrar los distintos espacios físicos. Esto se convierte en una necesidad importante para la generación de una solución tecnológica que permita realizar dicho proceso de forma automatizada, minimizando los tiempos de ingreso, realizando un control eficiente del personal debidamente autorizado y posibilitando además la generación de reportes actualizados.

Adicionalmente, se encuentra la necesidad de implementar un sistema de registro automático y sistematizado de la asistencia de los estudiantes a clases, ya que el método empleado para dicha tarea consiste en la recolección de firmas en formatos impresos, mientras que el sistema propuesto minimiza el uso de papel aportando al cuidado del medio ambiente, al mismo tiempo que permite la generación de un reporte más preciso, real y actualizado de la asistencia de los estudiantes, insumo importante para el tratamiento de la deserción estudiantil.

El desarrollo del proyecto se basa en el diseño de un prototipo de control de acceso que implemente de manera más rápida y sencilla una administración confiable, de manera que se puede obtener información precisa e instantánea sobre la ubicación de cada usuario (profesores, estudiantes), al mismo tiempo que se puede restringir el acceso no autorizado a las aulas de clases. Adicionalmente se pretende controlar de forma sistemática la toma de asistencia de estudiantes maximizando la eficiencia y mejorando la productividad con una administración segura.

\section{MARCO TEÓRICO DEL PROYECTO}

La automatización es el proceso de aplicación de diferentes técnicas para eliminar o disminuir la intervención del hombre en un proceso, con el fin de optimizar los tiempos y la calidad del mismo. Para implementar la automatización se requiere de diferentes disciplinas.

Las principales tecnologías sobre las cuales se basa el presente proyecto son RFID, ZigBee y Arduino. A continuación se realiza una breve descripción de cada una de éstas.

Identificación por Radio Frecuencia (RFID Radio Frequency IDentification): Se puede definir como un sistema remoto de almacenamiento y recuperación de datos. Se compone principalmente por una etiqueta o tag que permite el almacenamiento de datos, un lector que detecta y decodifica la información del tag y el software que interpreta dicha información (S. Delgadillo, J. Ortiz, 2011).

Sus inicios se remontan hacia los años 40's, durante la Segunda Guerra Mundial, cuando militares estadounidenses la emplean para diferenciar los aviones amigos de los enemigos. $\mathrm{Su}$ evolución pasa por la industria ferroviaria, la seguridad de materiales nucleares (años 60's y 70's) (J. Alvarado, 2008), y hacia los 80's en Europa la emplean para identificar ganado y en las cadenas de fabricación de la industria del automóvil. En los años 90's IBM logra integrar el sistema RFID en un solo chip electrónico. Seguidamente se ha diversificado el uso de la tecnología RFID en las industrias de servicio, compra y logística de distribución, fábricas y sistemas de flujo de metales. (V. J. Acevedo, A. García, J. S. Sandino, 2004).

En la actualidad esta tecnología tiene aplicaciones en seguridad, como las credenciales de identidad, peajes electrónicos, envío de paquetes, control de equipaje y de artículos para renta o préstamo, en la industria automotriz, en el sector agrícola y en el de administración de fauna y flora, entre otros. (J. Alvarado, 2008).

ZigBee: El nombre de este protocolo de comunicaciones tiene se origen en los patrones que emplean las abejas entre las flores para comunicarse cuando recogen el polen, evocando redes inalámbricas invisibles. (C. Ortega, D. Roque, L. Úbeda., 2008).

ZigBee surge por la demanda cada vez mayor de tecnologías inalámbricas sobre dispositivos de baja potencia, fiables y de baja tasa de datos. En octubre del 2002 fue creado el grupo de trabajo ZigBee Alliance, conformado por industrias como Mitsubishi, Philips y Motorola.

Este grupo desarrolló el estándar IEEE 802.15.4 sobre el cual se encuentra basada la tecnología, y en Mayo de 2003 el estándar fue terminado y publicado por IEEE. (A. Girón, 2012)

IEEE 802.15.4 precisa la capa física y de control de acceso al medio para redes inalámbricas de área personal cuyas tasas de transmisión de datos son bajas.

Su principal propósito fue proporcionar un estándar de comunicaciones de radio y bidireccional, con aplicación en dispositivos de automatización en el 
hogar, de edificio, control industrial, periféricos de PC y sensores médicos. De esta forma se da solución a las limitantes de Bluetooth.

ZigBee 1.0 fue su primera versión, liberada en Diciembre de 2004. Más adelante, en el año 2006, se realizaron revisiones al estándar 802.15.4 y al protocolo ZigBee en el 2007. [Cruz V. Enrique, Sánchez G. Carlos, 2009]

Arduino: Es una plataforma de hardware libre cuyo objetivo es facilitar la creación de prototipos electrónicos. Está basado en una placa con un microcontrolador, un entorno de desarrollo y un software de programación denominado Arduino basado en $\mathrm{C} / \mathrm{C}++$.

Se creó para artistas, diseñadores, aficionados y cualquiera interesado en crear entornos u objetos interactivos de manera rápida y a bajo costo.

Los programas hechos con Arduino se dividen en tres partes principales: estructura, valores (variables y constantes) y funciones.

Arduino puede tomar información del entorno a través de pines configurados como entradas desde toda una gama de sensores para ejercer control sobre luces, motores y otros actuadores.

El microcontrolador en la placa Arduino se programa mediante el lenguaje de programación Arduino (basado en Wiring) y el entorno de desarrollo Arduino (basado en Processing).

\section{DESARROLLO DEL PROYECTO}

El proyecto consiste en la identificación del usuario, su autenticación por parte del gestor del sistema y la activación del circuito electrónico de apertura de puertas o el registro de asistencia según sea el perfil del usuario. La lógica de operación del sistema propuesto se muestra en la figura 1.

En el diagrama de flujo ilustrado en la figura 1 se realiza el control de apertura de puertas y almacenamiento de los registros de la toma automática de asistencia a través de la validación de información, confrontando los datos leídos con los contenidos en una base de datos que contiene los horarios y docentes asignados a los diferentes recursos.

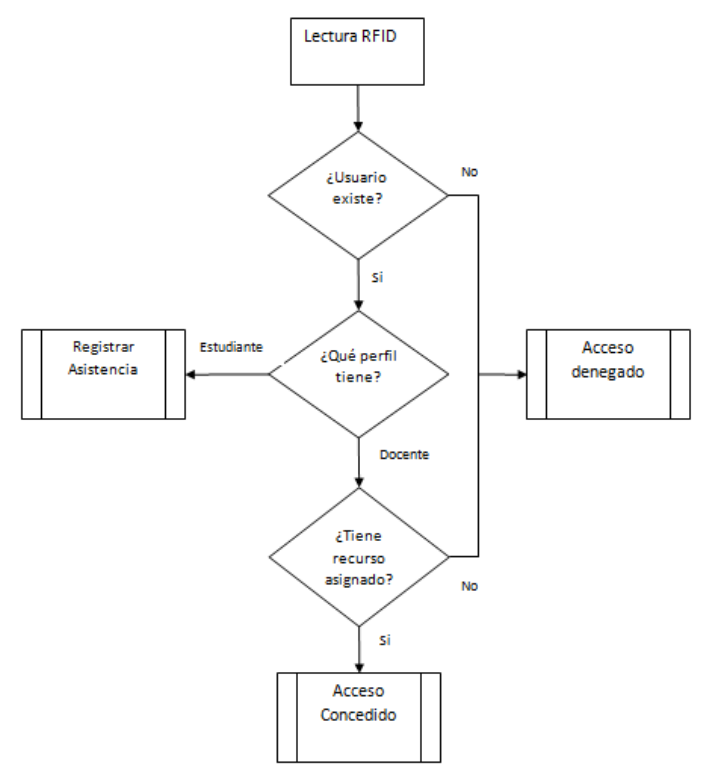

Fig. 1. Diagrama de flujo del software del sistema. Fuente: Elaboración propia.

\subsection{Estructura funcional del Sistema}

Teniendo como base la lógica de operación se procede a definir la estructura funcional del sistema. Ésta consta de una interfaz que permite la captura de la identificación del usuario, un controlador central, que es el encargado de realizar la conexión al servidor del sistema, el cual contiene la base de datos con los privilegios del usuario, y donde se registra la actividad (ya sea ingreso o toma de asistencia) y mensaje de respuesta al controlador central.

A partir de la estructura planteada, se definen los elementos principales del sistema, y se realiza un análisis de las diferentes tecnologías que podrían implementarse en cada etapa del prototipo, evaluando desempeño, flexibilidad y costo para todos los casos. De dicho análisis se decide emplear tecnología inalámbrica para la comunicación entre los puntos clientes ubicados en cada puerta de acceso. Adicionalmente se escoge la tecnología RFID (RadioFrequency IDentification) como interfaz usuario - sistema, el cual ofrece ciertas ventajas sobre otras tecnologías también utilizadas en sistemas similares, convirtiéndose en una herramienta clave y de éxito para funciones como almacenar un mayor número de información de forma segura, mayor control de productos etiquetados, facilidad de lectura, rapidez de respuesta entre otros. (S. Delgadillo, J. Ortiz, 2011; V. J. Acevedo, A. García, J. S. Sandino, 2004). 
En cuanto a la comunicación de tipo inalámbrica entre los controladores secundarios y el central se decide implementar módulos XBee, basados en el estándar ZigBee, que representan bajo consumo, economía, instalación y mantenimiento sencillo, alcances de comunicación ideal para propósitos de áreas medianas a pequeñas, entre otras características que lo consolidan como la mejor opción para la tarea a realizar.

Se complementa la estructura del sistema escogiendo el tipo de controladores a emplear y las conexiones que se requieren. La estructura definida se presenta en la figura 2.

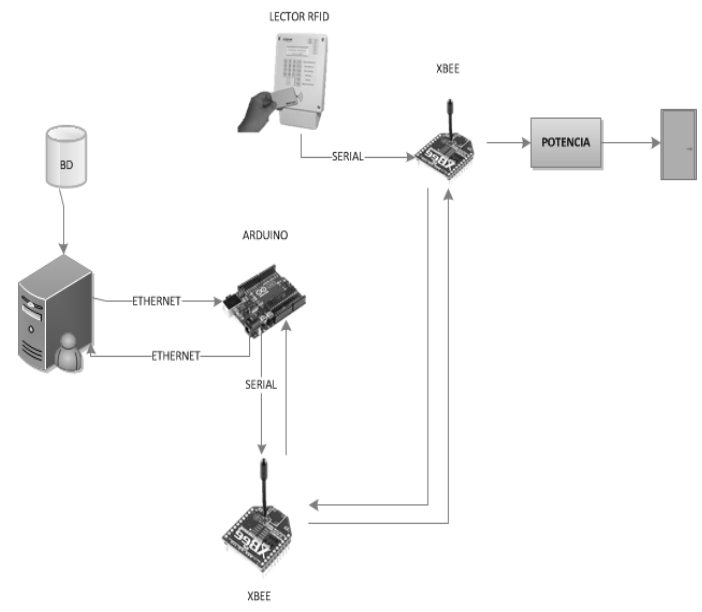

Fig. 2. Estructura Operacional del Sistema. Fuente: Elaboración propia.

El primer elemento es el dispositivo lector RFID encargado de leer los datos del usuario, operando en modo "escucha" por el cual se encuentra constantemente esperando un tag que para el caso opera de modo pasivo, es decir, el tag sólo funciona al ser energizado cuando se acerca al lector. De esta manera el tag envía su código de identificación del usuario al lector y éste último decodifica el dato recibido para transmitirlo a la siguiente etapa. (V. García, 2006; A. Gidekel. 2006).

El elemento siguiente es un circuito adaptador que convierte los niveles de voltaje RS232 a TTL, para poder acoplar la señal del puerto serial conectado al lector con el dispositivo electrónico de transmisión inalámbrica.

Los módulos XBee, son los dispositivos de interconexión inalámbrica entre lector $\mathrm{y}$ controlador central. El primer módulo transfiere mediante RF los datos adquiridos por el lector hacia otro módulo XBee que actúa como coordinador dentro la red.
Éste segundo dispositivo XBee está conectado a un módulo Arduino vía serial, que actúa en el sistema como controlador central. El Arduino, consta de un shield Ethernet para transferir los datos al servidor, que, como se mencionó anteriormente, corre el software para realizar las operaciones de comparación y registro.

El uso del arduino permite la ubicación del coordinador XBee en un punto estratégico para obtener mejores resultados en cuanto a topología y desempeño de la red XBee, debido a que éste no tiene que estar asociado de forma directa a una computadora que requiere de mayor espacio y tomas eléctricos cercanos para su funcionamiento, limitando de esta manera no solo la ubicación sino la independencia eléctrica del sistema ya que tanto el arduino como el módulo XBee pueden trabajar con baterías.

El microcontrolador PIC es el encargado de enviar la orden de activación a los actuadores requeridos y a su vez visualizar un mensaje sobre una LCD según la respuesta generada.

Posteriormente, se diseña el software que correrá sobre el servidor cuya finalidad será validar los datos recibidos de acuerdo a la lógica desarrollada, adaptando la estructura operacional del sistema a los protocolos del instituto, para poder cumplir con los lineamientos y políticas institucionales.

\subsection{Desarrollo de la etapa electrónica}

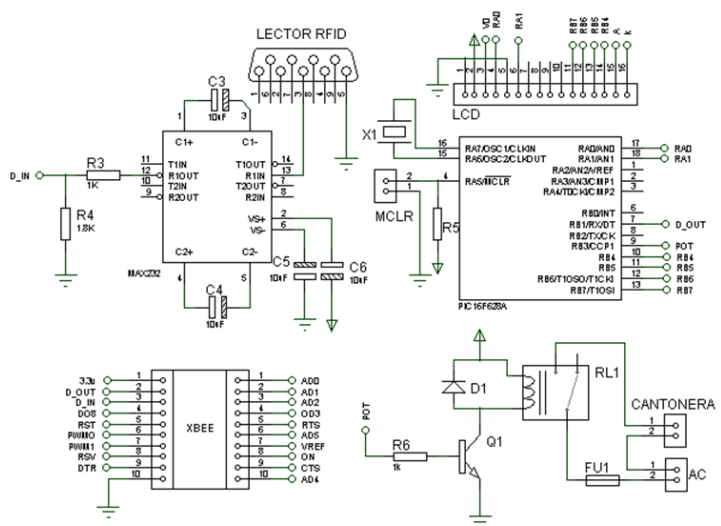

Fig. 3. Diagrama del circuito electrónico. Fuente: Elaboración propia.

En la figura 3 se muestra el circuito esquemático correspondiente a las tarjetas de adquisición de datos y control de acceso, que se ubicarían en cada una de las puertas de las aulas y laboratorios. Éstas constan de un puerto serial que brinda conexión entre el lector RFID y la tarjeta a través del circuito 
integrado MAX232 el cual adapta los niveles de voltaje RS232 a niveles que el XBee puede manejar. El XBee constituye el módulo de RF que envía el dato obtenido hacia el XBee coordinador. Luego se encuentra el módulo visualizador y de apertura de puerta constituido por un microcontrolador, una LCD y un circuito de potencia.

\subsection{Configuraciones y pruebas preliminares}

Para la configuración de los módulos XBee se utilizó la aplicación X-CTU de Digi, la cual brinda una interfaz gráfica sencilla para la realización de esta tarea, ya que los dispositivos XBee también pueden configurarse por comandos AT a través de hyperterminal o sobre cualquier aplicación de interfaz serial. Cada módulo se configura de acuerdo a la función que desempeña dentro de la red, las cuales pueden ser coordinador, router o dispositivo final, en la figura 4 se muestra la interfaz de la aplicación y se resaltan los parámetros más importantes.

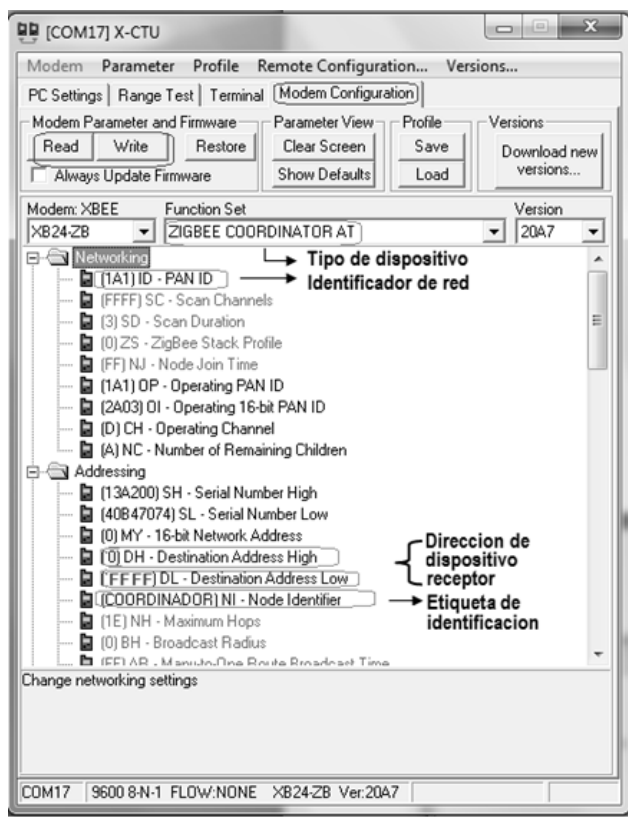

Fig. 4. Programación módulos XBee. Fuente: Elaboración propia.

Las pruebas preliminares consisten en comprobar la comunicación entre dos módulos XBee. Para dicha prueba se conectó el primer módulo vía puerto serial a un PC mediante el programador de XBee, mientras que el otro módulo se conectó a un dispositivo de control de Arduino y este último al pc. Se logra establecer la comunicación bidireccional entre ambos módulos XBee, enviando pequeñas tramas de palabras e incluso un archivo de 195 Kilobytes.

Adicionalmente la programación del Arduino requirió algunas librerías especiales para la comunicación serial con el XBee, empleando el programador Arduino.

Luego se procede a realizar nuevas pruebas donde se realicen las conexiones punto a multipunto entre módulos XBee, de manera que se puedan tener módulos para los distintos puntos de acceso.

Finalmente se incorpora la etapa de transmisión inalámbrica con los circuitos electrónicos de control de apertura y con el Arduino conectado al servidor. De esta manera se puede comprobar globalmente el funcionamiento del sistema.

\section{RESULTADOS}

Como resultado cuando un docente desea ingresar a un salón de clases, sala de informática, laboratorio $\mathrm{u}$ otro recurso físico del instituto para desarrollar su clase, debe presentar su etiqueta o tag RFID ante el lector ubicado junto a la puerta de acceso. El lector toma la identificación del tag y lo envía al módulo XBee, y éste a su vez al módulo Arduino para transferir la petición al computador que actúa como servidor, donde se encuentra la aplicación y la base de datos de los usuarios, allí se verifica si el docente tiene asignado el recurso físico en el horario y fecha del momento, envía la respuesta de regreso al Arduino, éste a través de los módulos XBee al microcontrolador de la puerta señalada, el cual da la orden al circuito electrónico de apertura de puertas que desplegará un mensaje en una pantalla LCD donde aparecerá Acceso Denegado o Acceso Autorizado, según sea el caso. $\mathrm{Si}$ el docente está autorizado la cerradura electrónica se abrirá y permitirá ingresar al docente y sus estudiantes.

Los estudiantes, por su parte, al ingresar también deben presentar su tag para identificarse y el sistema de control de asistencia coloca automáticamente las fallas o asistencias de cada uno, teniendo en cuenta el horario establecido, todo sobre la misma infraestructura tecnológica descrita en el proceso anterior. Con estos se posibilita la generación de reportes reales los cuales serían empleados por el área encargada para el seguimiento a los estudiantes y así reducir el nivel de ausentismo en la institución. 
Dentro de las pruebas del funcionamiento del sistema se realizaron lecturas de tag validados con rol de docente y estudiante obteniendo la identificación correcta y ejecución de la respuesta configurada, para el caso de docentes y estudiantes se verificaron dos situaciones en el primer caso se acercó un tag de un docente que no estaba asignado al recurso a lo cual la respuesta fue acceso denegado, y en el segundo un tag de un docente con recurso asignado realizando la apertura de la puerta y registrando el ingreso del docente. Para el caso de estudiante se validó la asistencia al aula mediante su tag de identificación y el acceso denegado debido al no ingreso inicial de un docente al aula.

En caso de fallas eléctricas la apertura de las puertas puede funcionar de modo tradicional sin causar traumatismos en el desarrollo de clases. Para el caso en que el docente no posea su carné para entrar debido a pérdida del mismo, se podrá solicitar al administrador de recursos un tag provisional al cual se le configura la identificación del docente durante la jornada correspondiente. Adicionalmente, si el estudiante no presenta su carné, puede solicitar al docente el registro por sistema de la asistencia de forma manual, con solo ingresar a la plataforma con su usuario y contraseña y digitar la identificación del estudiante para el registro en la clase específica.

El diseño e implementación del sistema de control de acceso con RFID y XBee a los recursos físicos del ITSA permite implementar una nueva estrategia para el desarrollo de las actividades cotidianas de la academia contribuyendo con mejorar el rendimiento de los procesos a partir del uso y apropiación de las tecnologías inalámbricas y de identificación. A nivel investigativo, el proyecto conlleva a fortalecer la capacidad científica tecnológica de los desarrolladores y aprovechar las destrezas de las distintas disciplinas de los participantes del proyecto para desarrollar las fases de este, además genera nuevos conocimientos en los integrantes del equipo en la aplicación de tecnologías inalámbricas emergentes, particularmente RFID y Zigbee.

\section{CONCLUSIONES}

Con el desarrollo y ejecución de este proyecto se logra obtener un prototipo piloto con el fin de sistematizar el proceso de acceso a aulas, salas de informáticas y laboratorios, y toma de asistencia de clases en el Instituto Tecnológico de Soledad
Atlántico, aplicando tecnologías cuya utilización en el país aumenta día a día y tiende a seguir expandiéndose. Esto ubica a la institución en un mejor nivel tecnológico y a los participantes del proyecto les brinda una nueva competencia en la aplicación de tecnologías inalámbricas.

El sistema completo consiste en el desarrollo de software que soporta toda la gestión de recursos y asistencia, los equipos RFID y demás hardware que controlan al acceso a los espacios físicos y la comunicación entre los puntos de acceso en cada salón y el controlador central (Arduino) mediante ZigBee.

La prueba piloto ha arrojado resultados satisfactorios, ya que se puede activar el circuito electrónico de apertura de puertas los punto de acceso al identificarse con el tag en el lector indicado o ingresando por sistema. Adicionalmente la comunicación multipunto de los equipos XBee funciona efectivamente y de forma transparente a los usuarios, lográndose fácil instalación, escalabilidad y mantenimiento de la red. Sin embargo se hace necesario la realización de pruebas a gran escala, es decir, establecer un mayor número de terminales de control y de esta manera evaluar el comportamiento del sistema. Para esto se ha planificado el establecimiento de diferentes controladores asociados a áreas de control de hasta 5 terminales, los cuales estarán conectados a diferentes PAN ID.

\section{REFERENCIAS}

S. Delgadillo, J. Ortiz. (2011). "Diseño de un Sistema de Control de Acceso Mediante Tecnología RFID con Implementación de un Servidor Web embebido en un PIC" Tesis de Ingeniería en Comunicaciones y Electrónica, Universidad Autónoma de Zacatecas, Zacatecas, México.

J. Alvarado. (2008). "Sistema de Control de Acceso con RFID" Tesis de Maestría en Ciencias, Departamento de Ingeniería Eléctrica, Centro de Investigación y de Estudios Avanzados del Instituto Politécnico Nacional, México D.F., México.

V. J. Acevedo, A. García, J. S. Sandino.(2004) "Sistema de Registro y Control de Salida de Elementos Mediante Dispositivos RFID" Trabajo de Grado de Ingeniero Electrónico, Departamento de Electrónica, Facultad de Ingeniería, Pontificia Universidad Javeriana, Bogotá D.C., Colombia, Noviembre. 
C. Ortega, D. Roque, L. Úbeda. (2008). "Zigbee: El nuevo estándar global para la Domótica e Inmótica". Universidad Nacional de Ingeniería. Facultad de Electrotecnia y Computación, Managua, Nicaragua, Julio.

A. Girón. (2012) "Desarrollo e implementación de una red de sensores Zigbee mediante el dispositivo Xbee de Digi". Titulación en Ingeniería en Automática y Electrónica Industrial. Escola Tecnica Superior Enginyeria - Universitat Rovira I Virgili. Catalans, España.

Cruz V. Enrique, Sánchez G. Carlos. (2009) "Comunicación entre microcontroladores bajo el protocolo Zigbee". Instituto Politécnico Nacional. México.

Arduino, Arduino, Fecha de Acceso: 23/06/2014, http://www.arduino.cc/es/
Arduino, Referencia del Lenguaje, Fecha de Acceso: $23 / 06 / 2014$ http://arduino.cc/es/Referen ce/HomePage

V. García. (2006) "Estudio de la identificación por radio-frecuencia (RFID) y desarrollo de software relacionado con el control de la cadena de suministro" Proyecto de fin de carrera, Escuela Técnica Superior de Ingeniería de Telecomunicación, Universidad de Málaga, Málaga, España.

A. Gidekel. (2006). RFID Introducción a la Identificación por Radio Frecuencia, 1ra Ed, Argentina, Telectrónica Codificación S.A., pp. 7-31.

Digi, $X-C T U$ (XCTU) software, Acceso: $02 / 12 / 2013$,

http://www.digi.com/support/kbase/ kbaseresultdetl?id=2125. 